\title{
Internal Carotid Artery and the Proximal M1 Segment Are Optimal Targets for Mechanical Thrombectomy
}

\author{
Niko Sillanpää d Sara Protto ${ }^{d} \quad J u k k a ~ T$. Saarinen $^{c} \quad$ Juha-Pekka Pienimäki ${ }^{d}$ \\ Janne Seppänen $^{d}$ Heikki Numminen $^{a}$ Harri Rusanen $^{b}$ \\ a Department of Neurology, Tampere University Hospital, Tampere, ${ }^{b}$ Department of \\ Neurology, Oulu University Hospital, Oulu, ' Vaasa Central Hospital, Vaasa, and d Medical \\ Imaging Center, Tampere University Hospital, Tampere, Finland
}

\section{Keywords}

Intravenous thrombolysis - Ischemic stroke - Mechanical thrombectomy · Revascularization · Stent retriever

\begin{abstract}
Background and Purpose: Mechanical thrombectomy (MT) is an established treatment of acute anterior circulation stroke caused by large vessel occlusion (LVO). We compared the clinical outcome (3-month modified Rankin Scale, mRS) in hyperacute ( $<3 \mathrm{~h}$ from the onset of symptoms) ischemic stroke between an MT and an intravenous thrombolysis (IVT) cohort in proximal (ICA and the proximal M1 segment of the middle cerebral artery) and distal (the distal M1 and the M2 segment) LVOs. Methods: We prospectively reviewed 67 patients who underwent MT with newer-generation stent retrievers. The IVT cohort consisted of 98 patients who received IVT without MT. We recorded baseline clinical, procedural and imaging variables, technical outcome, 24-h imaging outcome, and the clinical outcome. Differences between the groups were studied with theoretically appropriate statistical tests and binary logistic regression analysis. Results: The proportion of patients who had a proximal LVO and experienced good $(m R S \leq 2)$ or excellent $(m R S \leq 1)$ clinical outcome was significantly larger in the MT group (62 vs. $7 \%, p<0.001 ; 47$ vs. $3 \%, p<0.001$, respectively). In a regression model including relevant confounding variables, good clinical outcome was seen significantly more often among patients with proximal occlusions (OR $=6.0, \mathrm{CI} 95 \% 1.9-18.3, p=0.002$ ). In a similar model, no statistically significant differences were observed in patients with more distal occlusions. Conclusions: MT is superior to IVT in achieving good clinical outcome in hyperacute anterior circulation stroke in the most proximal occlusions (ICA and proximal M1 segment). In the distal $\mathrm{M} 1$ and $\mathrm{M} 2$ segments neither of these therapies clearly outperforms the other.




\section{Introduction}

Mechanical thrombectomy (MT) is a safe and efficient treatment for acute ischemic stroke caused by large vessel occlusion (LVO) with sufficiently small infarct volume on admission imaging as established by several recent randomized studies [1-5]. Based on these trials there is convincing evidence that MT yields better results compared to revascularization with intravenous thrombolysis (IVT) only if the occlusion is located in the distal internal carotid artery (ICA) including the different conformations carotid I, L and T, or the M1 segment of the middle cerebral artery (MCA). It is still unclear if MT outperforms IVT in the M2 segment because of the small number and heterogeneousness of patients with M2 occlusions in the randomized trials and other previous research [6-8]. Further, according to a recent recommendation and previous reports, the proximal and distal halves of the M1 segments should be examined separately because these vessel subsegments have differing properties that seem to influence the potential for good clinical outcome and the efficacy of revascularization therapies [8-12].

We compared the clinical outcome measured with 3-month modified Rankin Scale (mRS) in hyperacute ( $<3 \mathrm{~h}$ from the onset of symptoms) ischemic stroke between an MT and an IVT cohort in different sites of occlusion: ICA, the proximal M1 (M1P), the distal M1 (M1D), and the M2 segment of the MCA. The first two sites were considered proximal LVOs and the latter two distal LVOs.

\section{Methods}

Overview, Participants, and Variables

We prospectively collected and analyzed the clinical and imaging data of 130 consecutive patients, who were admitted to Tampere University Hospital from January 2013 to December 2014 because of acute ischemic stroke symptoms and who underwent clinical and imaging evaluation and proceeded to digital subtraction angiography (DSA) with an intention to perform MT. The inclusion criteria were occlusion of ICA (the intracranial part with possible extension to the extracranial part) and/or the M1 or M2 segment of the MCA, MT with a stent retriever, and onset-to-imaging time $3 \mathrm{~h}$ or less. Sixty-seven patients met these criteria. An anterior circulation occlusion IVT-only cohort was used as a control group. This cohort has been extensively characterized in our previous report [12]. Ninety-eight from 105 patients remained in this group after patients with occlusions distal to the M2 segment were removed. The IVT-only cohort includes all patients treated with IVT from 2004 to 2007 for anterior circulation stroke and who had a clot visible in CT angiography (CTA). Starting from 2008 with the advent of the first generation MT devices we began to treat selected patients with MT. By 2012, we had established a stroke protocol where all anterior circulation stroke patients being evaluated for IVT were also evaluated for MT if there was a clot visible in CTA. The inclusion criteria to treat LVO (ICA and the M1 and M2 segments) with MT paralleled those of IVT apart from the time limit from the onset of symptoms that was $<12 \mathrm{~h}$ with MT, and therapy-related differences in contraindications. The prestroke mRS was required to be $\leq 2$. In the current study, only onset-to-imaging times $<3 \mathrm{~h}$ were included to maintain congruence with the IVT-only cohort.

The initial imaging evaluation consisted of non-contrast-enhanced computed tomography (NCCT), CTA, and CT perfusion (CTP). CTP could be dropped to save time at discretion of the attending neurologist. The selection of patients as candidates for MT was based on absence of extensive irreversible ischemic changes (frank hypodensity spanning more than one-third the volume of MCA vascular territory) and hemorrhage in NCCT, presence of a proximal clot in CTA, and evaluation of the amount of salvageable tissue in CTP imaging (if available).

Baseline clinical characteristics included age, sex, and clinical risk factors for ischemic stroke (hypertension, diabetes, coronary heart disease, atrial fibrillation). These data were collected from the patient records. National Institutes of Health Stroke Scale (NIHSS) score at the presentation, time from symptom onset to imaging and recanalization of the occluded vessel, the duration of the procedure, the Thrombolysis in Cerebral Infarction (TICI) score, and possible complications had been prospectively stored to a specifically devised questionnaire. A follow-up NCCT was performed $24 \mathrm{~h}$ after revascularization. Hemorrhagic complications and cerebral edema (COED) were classified according to the SITS-MOST criteria. 
Reperfusion was evaluated with TICI, which was scored from the final DSA control runs of the intervention. The clinical outcome measure was mRS, evaluated 3 months after the stroke based on a follow-up visit to a neurologist or a phone interview by a neurologist. The imaging outcome measure was infarct volume evaluated $24 \mathrm{~h}$ after the intervention.

All procedures performed were in accordance with the ethical standards of the institutional and/or national research committee. For this type of study, formal consent is not required.

\section{Revascularization Therapies}

MT was performed using a bi-axial system consisting of an 8-Fr or 9-Fr guiding catheter with a tip balloon and coaxially a $0.021^{\prime \prime}$ micro-catheter or a tri-axial system consisting of an 8-Fr guiding catheter, a distal access catheter through which a micro-catheter was inserted with the aid of a $0.014^{\prime \prime}$ micro-guidewire. The micro-catheter was navigated through the occluded segment of the artery, and a suitable stent retriever was positioned trough the micro-catheter to the site of the thrombus and deployed. The stent was left in place for $4 \mathrm{~min}$ and then retrieved and at the same time the guiding or the intermediate catheter was aspirated forcefully. The same procedure was repeated until satisfactory circulation was restored. Different stent retrievers were used based on the preference and judgment of the operator. TREVO ${ }^{\circledR}$ (Stryker Neurovascular/Concentric Medical, Mountain View, CA, USA) was used in 40\% of cases, CAPTURE LPTM (eV3/ COVIDIEN/Medtronic, Santa Rosa, CA, USA) in 40\%, ERIC ${ }^{\circledR}$ (MicroVention, Tustin, CA, USA) in 9\%, Aperio ${ }^{\circledR}$ (Acandis, Pforzheim, Germany) and REVIVE ${ }^{\circledR}$ (Codman \& Shurtleff, Raynham, MA, USA) in 1\%, respectively, and in $10 \%$ of cases multiple device types were used. The diameters used varied from 3 to $6 \mathrm{~mm}$ with a $4-\mathrm{mm}$ device being most commonly used. IVT (Actilyse ${ }^{\circledR} 0.9 \mathrm{mg} / \mathrm{kg}$, Boehringer-Ingelheim, Ingelheim, Germany) was administered based on the judgment of the attending stroke neurologist and possible contraindications. Actilyse ${ }^{\circledR}$ bolus was given on the CT table. Actilyse ${ }^{\circledR}$ drip was continued until groin puncture or full dose.

\section{Imaging Parameters}

The imaging parameters are characterized in the Appendix.

\section{Image Analysis}

The details of image analysis and validation of the measurements are described in the Appendix.

\section{Statistics}

The data were analyzed with SPSS version 20 (SPSS Inc., Chicago, IL, USA). Group comparisons were performed by using the Student $t$ test, the $\chi^{2}$ test, the Fisher exact test, and the Mann-Whitney U test. Binary logistic regression analyses using good and excellent clinical outcomes as dependent variables were performed in order to control for the effect of differences in NIHSS and, when analyzing proximal LVOs, the presence of coronary artery disease and/or atrial fibrillation, or when analyzing distal LVOs, the presence of chronic hypertension, the onset-to-imaging time and the clot burden score between the MT and IVT-only groups. These variables were selected based on the results of univariate analyses and theoretical considerations. Odds ratio (OR) with 95\% confidence interval (CI) was calculated for each covariate. Patients who had 3 -month mRS $\leq 2$ were considered to have experienced good clinical outcome and those with mRS $\leq 1$ excellent outcome. Occlusions in ICA and/or M1P were defined as proximal and those in M1D and M2 segment as distal. A $p$ value $<0.05$ was considered statistically significant.

\section{Results}

\section{Baseline Characteristics}

The inclusion criteria were met by 67 patients in the MT group and 98 patients in the IVT-only group. In the MT cohort 38 patients were excluded because of onset-to-imaging time (time to treatment decision making) longer than $3 \mathrm{~h} ; 8$ patients had posterior circulation strokes, 1 patient had occlusion of the A3 segment of the anterior cerebral artery, and another clot in the M3 segment. In 15 cases, MT was not performed either because the clot had dissolved or there was no access to the thrombus. The baseline and admission imaging characteristics for all patients and both groups are summarized in the Appendix. In one case, 
Fig. 1. Clot locations in the MT and IVT-only groups. ICA, internal carotid artery; M1, middle cerebral artery segment 1 ; M2, middle cerebral artery segment 2 .
Sillanpää et al.: Internal Carotid Artery and the Proximal M1 Segment Are Optima Targets for Mechanical Thrombectomy

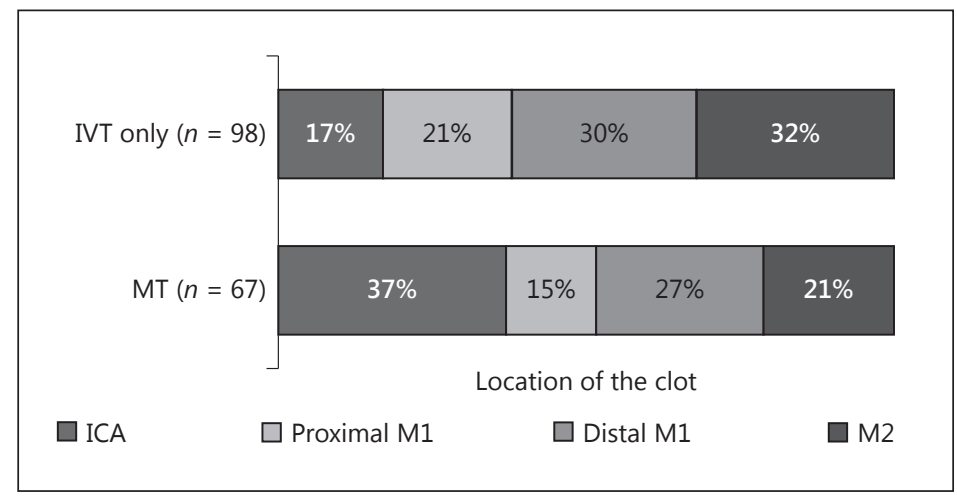

NIHSS could not be scored reliably because of sedation during transportation. CTP was successfully obtained from 42 patients (63\%) in the MT group and 76 patients $(77 \%)$ in the IVT-only group. The reperfusion outcome was TICI $2 \mathrm{~b}$ or 3 in 59 out of 67 cases (88\%). The results were nonsignificantly above average in the M1P segment (100\%) and worse in the M2 segment (79\%). The median duration of the procedure was $30 \mathrm{~min}$ (IQR $28 \mathrm{~min}$ ) and the median onset-to-reperfusion time was $180 \mathrm{~min}$ (IQR $75 \mathrm{~min}$ ). The median interval between admission imaging and groin puncture was $48 \mathrm{~min}$ (IQR $50 \mathrm{~min}$ ). The distribution of locations of the clots is depicted in Figure 1. The proportion of patients with distal thrombi was larger in the IVT-only group (48 vs. 62\%). Thus, proximal (ICA and M1P) and distal (M1D and M2) LVOs were studied separately.

Among those with proximal LVOs, there were no significant differences between the groups in age or the other established stroke risk factors apart from there being significantly larger number of atrial fibrillation in the MT group and ischemic heart disease in the IVT-only group (Table 1). Those who received only IVT had significantly smaller lesions on mean transit time (MTT) maps.

Among those with distal LVOs, the strokes were less severe in the IVT-only group based on NIHSS, and the clot burden and the sizes of the perfusion defects on MTT maps were correspondingly significantly smaller (Table 1 ). There were no statistically significant differences in onset-to-imaging times.

\section{Outcomes in Different Sites of Occlusion}

The distributions of the 3-month mRS scores in both groups in different sites of occlusion are depicted in Figures 2 and 3. The proportion of patients who had a proximal LVO and experienced good or excellent clinical outcome was significantly larger in the MT group (62 vs. 7\%, $p<0.001 ; 47$ vs. $3 \%, p<0.001$, respectively; Tables 2,3 ). The results were similar when ICA and M1P occlusions were analyzed separately. Binary logistic regression analyses with good or excellent clinical outcome as dependent variables were performed to control for potential confounding variables. MT increased the odds of good clinical outcome 6-fold among patients with proximal LVOs (OR $=6.0$, CI 95\% 1.9-18.3, $p<0.001$; Table 2). Again, the results were similar with borderline significance when ICA and M1P occlusions were analyzed separately $(\mathrm{OR}=4.9$, CI 95\% 0.77-31.5, $p=0.09$; and OR = 11.9, CI 95\% 1.6-90.1, $p=0.02$, respectively) or excellent clinical outcome was the outcome measure (OR = 28.0, CI 95\% 3.4-233.8, $p<0.001$; Table 3). Mortality was significantly higher in the IVT-only group (12 vs. $32 \%, p=0.04$; Table 1 ). Those who received IVT-only to treat a proximal LVO had almost triple the mean infarct volume (35 vs. $96 \mathrm{~cm}^{3}, p=0.003$; Table 1) at $24 \mathrm{~h}$. Linked to the larger infarct volumes, these patients more often suffered from COED grade 2 or 3 postinfarct edema ( 24 vs. 53\%, $p=0.01$; Table 1 ). 

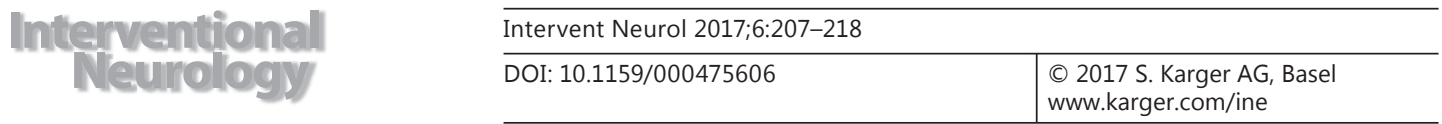

Sillanpää et al.: Internal Carotid Artery and the Proximal M1 Segment Are Optima

Targets for Mechanical Thrombectomy

Table 1. Baseline and admission imaging characteristics and the clinical and imaging outcomes of patients with proximal and distal large vessel occlusions in the MT and IVT-only groups

\begin{tabular}{|c|c|c|c|c|c|c|}
\hline \multirow[t]{2}{*}{ Characteristic } & \multicolumn{3}{|c|}{ ICA and proximal M1 segment } & \multicolumn{3}{|c|}{ Distal M1 and M2 segments } \\
\hline & $\begin{array}{l}\text { MT } \\
(n=35)\end{array}$ & $\begin{array}{l}\text { IVT only } \\
(n=38)\end{array}$ & $p_{1}$ & $\begin{array}{l}\text { MT } \\
(n=32)\end{array}$ & $\begin{array}{l}\text { IVT only } \\
(n=60)\end{array}$ & $p_{2}$ \\
\hline Age, years & $67.4 \pm 9.4$ & $65.7 \pm 15.2$ & 0.64 & $68.4 \pm 10.4$ & $70.7 \pm 12.8$ & 0.21 \\
\hline Male sex & $18(51)$ & $27(71)$ & 0.09 & $12(38)$ & $28(47)$ & 0.51 \\
\hline NIHSS before treatment & $14.5[5]$ & $17.5[7]$ & 0.12 & $14[6]$ & $11[10]$ & 0.01 \\
\hline ASPECTS score at admission NCCT & $9[2]$ & $9[3]$ & 0.66 & $10[1]$ & $10[2]$ & 0.68 \\
\hline ASPECTS score at admission MTT & $1[2]$ & $3[4]$ & 0.001 & $3.5[(5]$ & $6[3]$ & 0.04 \\
\hline ASPECTS score at admission CBV & $6.5[6]$ & $7[5]$ & 0.43 & $8.5[(3]$ & $8[3]$ & 0.53 \\
\hline Onset to imaging time, $\min$ & 94 [79] & $87[40]$ & 0.22 & $75[58]$ & $92[31]$ & 0.04 \\
\hline Clot burden score & $4[3]$ & $4[2]$ & 0.27 & $6[2]$ & $8[3]$ & 0.01 \\
\hline Collateral score & $1[2]$ & $1[3]$ & 0.91 & $2[2]$ & $2[2]$ & 0.90 \\
\hline Hypertension & $20(57)$ & $22(58)$ & 0.95 & $15(47)$ & $40(67)$ & 0.08 \\
\hline Diabetes & $3(7)$ & $8(21)$ & 0.14 & $7(22)$ & $8(13)$ & 0.38 \\
\hline Atrial fibrillation & $19(54)$ & $12(32)$ & 0.05 & $18(56)$ & $25(42)$ & 0.20 \\
\hline Coronary artery disease & $6(17)$ & $16(42)$ & 0.01 & $5(16)$ & $18(30)$ & 0.21 \\
\hline ASPECTS score at 24-h CT & $8[4]$ & $4[5]$ & 0.001 & $9[3]$ & $8[4]$ & 0.17 \\
\hline Total infarct volume at $24 \mathrm{~h}, \mathrm{~cm}^{3}$ & $4.5[45.7]$ & $59.4[153.1]$ & 0.001 & $2.5[24.3]$ & $8.3[38.7]$ & 0.32 \\
\hline Hemorrhagic complication at $24 \mathrm{~h}$ & $5(15)$ & $4(11)$ & 0.73 & $4(13)$ & $3(5)$ & 0.23 \\
\hline Major space-occupying effect (PH2 or PHr2) & $2(6)$ & $2(5)$ & 0.93 & $2(6)$ & $1(2)$ & 0.28 \\
\hline Postinfarct edema COED2 or COED3 & $8(24)$ & $19(53)$ & 0.01 & $6(19)$ & $10(17)$ & 0.78 \\
\hline Three-month mortality & $4(12)$ & $12(32)$ & 0.04 & $4(13)$ & $2(3)$ & 0.18 \\
\hline
\end{tabular}

Data are presented as mean \pm SD, $n(\%)$, or median [IQR]. P1, P2: $p$ values between groups; ASPECTS: Alberta Stroke Program Early CT Score; CBV, cerebral blood volume; COED, cerebral edema; MTT, mean transit time; NCCT, non-contrast-enhanced computed tomography; NIHSS, National Institutes of Health Stroke Scale; PH, parenchymal hemorrhage; PHr, parenchymal hemorrhage remote.

There were no statistically significant differences in clinical outcome between the MT and IVT-only groups when distal LVOs were considered after controlling for confounders (Tables 2, 3). However, there was a trend toward patients treated with MT for an M2 occlusion more often experiencing unfavorable clinical outcome (Fig. 3; Tables 2, 3). This difference was statistically significant ( $p=0.03$ ) before confounding was accounted for.

Sixty-three percent ( 42 of 67 ) of the patients who underwent MT received IVT before the procedure. Twenty-six of these (62\%) experienced good clinical outcome compared to 12 patients (48\%) among those without IVT ( $p=0.27)$.

\section{Discussion}

We compared the 3-month clinical outcome of MT performed with third-generation stent retrievers and IVT only in the treatment of hyperacute ischemic stroke in different sites of occlusion: ICA, the M1P, the M1D, and the M2 segments of the MCA. Our study confirms the findings of the recent randomized trials $[1-5,13,14]$ that MT is clearly superior to IVT in the treatment ICA and M1P occlusions (proximal LVOs). Only 12 and 24\%, respectively, of patients who received only IVT experienced good clinical outcome ( $\mathrm{mRS} \leq 2$ ) compared to 54 and $80 \%$ among those treated with MT. However, we did not find a significant difference in favor of either revascularization therapy in the treatment of M1D or M2 occlusions (distal LVOs). 
Sillanpää et al.: Internal Carotid Artery and the Proximal M1 Segment Are Optima Targets for Mechanical Thrombectomy

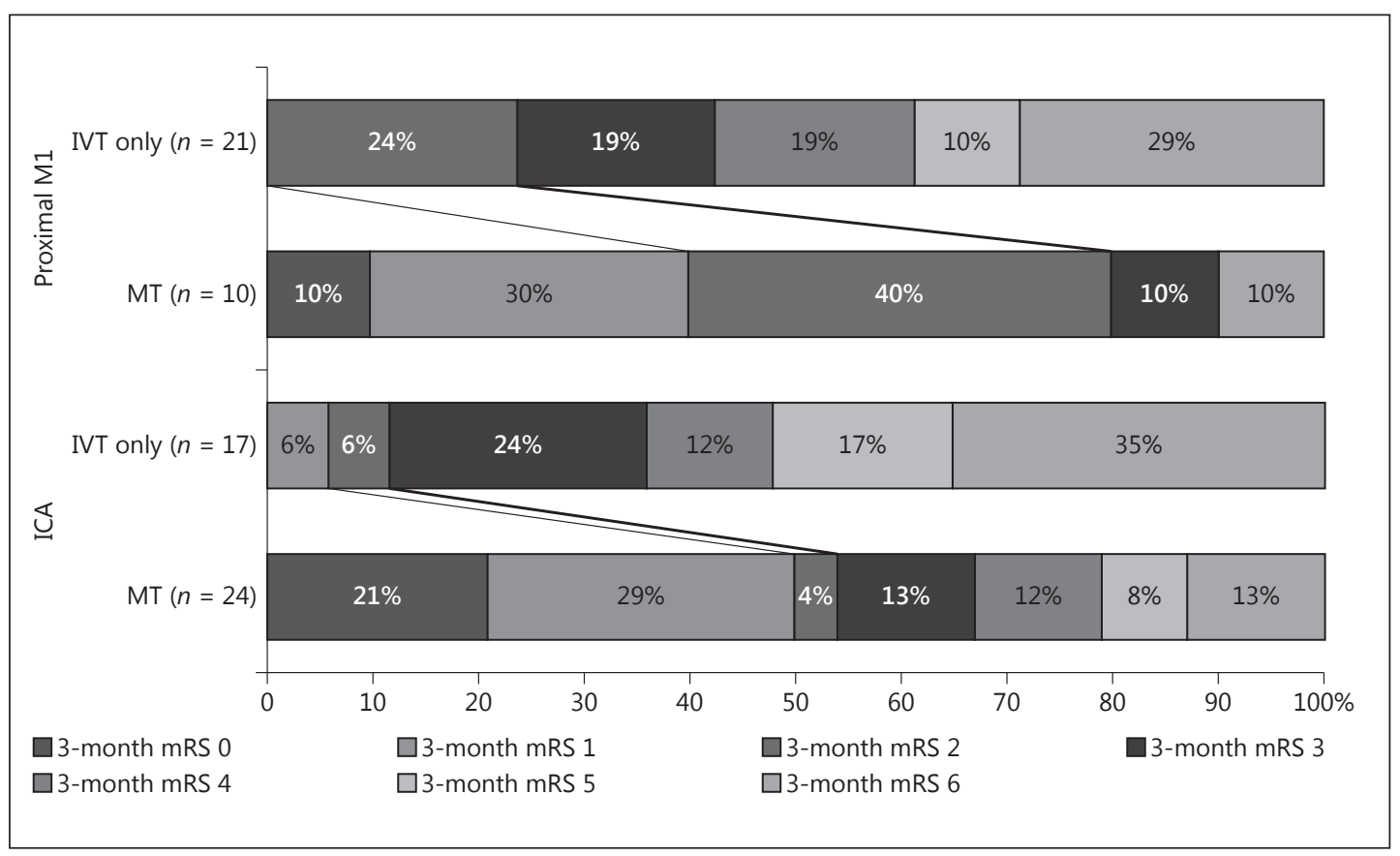

Fig. 2. The distribution of 3-month modified Rankin Scale (mRS) in the MT and IVT-only groups in proximal occlusions. The thick line indicates the division between functional independence ( $\mathrm{mRS} \leq 2$, left hand side) and dependence (right hand side) and the thin line demarcates those with excellent clinical outcome (mRS $\leq 1$, left hand side).

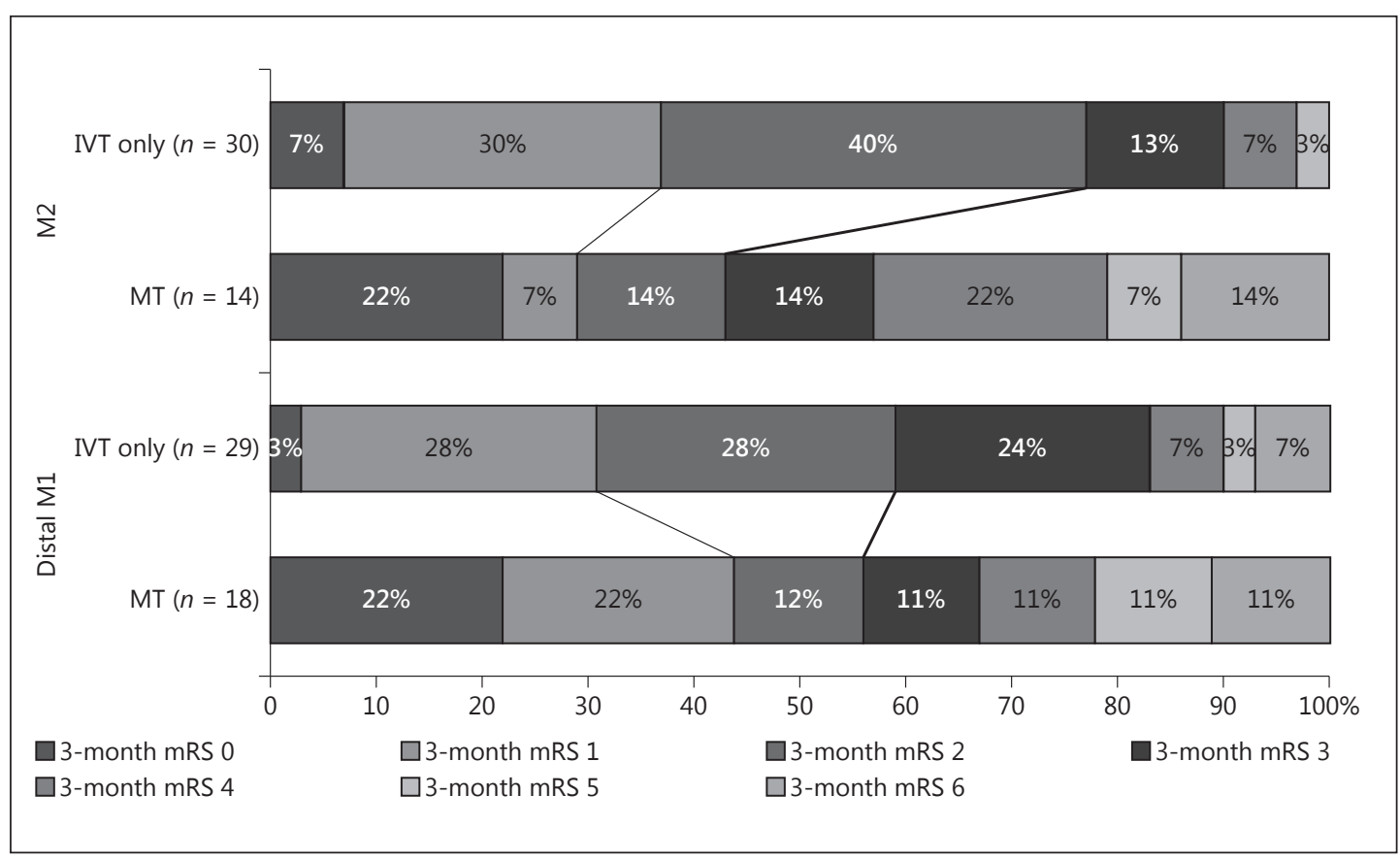

Fig. 3. The distribution of 3-month modified Rankin Scale (mRS) in the MT and IVT-only groups in distal occlusions. The thick line indicates the division between functional independence ( $\mathrm{mRS} \leq 2$, left hand side) and dependence (right hand side), and the thin line demarcates those with excellent clinical outcome ( $\mathrm{mRS} \leq 1$, left hand side). 
Table 2. Good clinical outcome in the MT and IVT-only groups

\begin{tabular}{lrrrrrr}
\hline Clot location & \multicolumn{1}{l}{$\begin{array}{l}\text { MT } \\
\text { mRS } \leq 2\end{array}$} & $\begin{array}{l}\text { IVT only } \\
\text { mRS } \leq 2\end{array}$ & \multicolumn{1}{c}{$p_{1}$} & Odds ratio & 95\% CI & $p_{2}$ \\
\hline ICA & $13(54 \%)$ & $2(12 \%)$ & 0.008 & 4.9 & $0.77-31.5$ & 0.09 \\
Proximal M1 & $8(80 \%)$ & $5(24 \%)$ & 0.006 & 11.9 & $1.6-90.1$ & 0.02 \\
Distal M1 & $10(56 \%)$ & $17(59 \%)$ & 0.84 & 1.0 & $0.26-3.7$ & 0.96 \\
M2 & $6(43 \%)$ & $23(77 \%)$ & 0.03 & 0.30 & $0.05-1.9$ & 0.20 \\
ICA and proximal M1 & $21(62 \%)$ & $7(18 \%)$ & $<0.001$ & 6.0 & $1.9-18.3$ & 0.002 \\
Distal M1 and M2 & $16(50 \%)$ & $40(69 \%)$ & 0.12 & 0.54 & $0.16-1.5$ & 0.24 \\
\hline
\end{tabular}

P1, $p$ value between groups; P2, significance of the covariate in binary logistic regression model; CI, confidence interval; ICA, internal carotid artery; M1, middle cerebral artery segment 1; M2, middle cerebral artery segment 2 .

Table 3. Excellent clinical outcome in the MT and IVT-only groups

\begin{tabular}{lrccccc}
\hline Clot location & $\begin{array}{l}\text { MT } \\
\text { mRS } \leq 1\end{array}$ & $\begin{array}{l}\text { IVT only } \\
\text { mRS } \leq 1\end{array}$ & $p_{1}$ & Odds ratio & $95 \%$ CI & $p_{2}$ \\
\hline ICA & $12(50 \%)$ & $1(6 \%)$ & 0.005 & 9.0 & $0.9-89.8$ & 0.06 \\
Proximal M1 & $4(40 \%)$ & 0 & 0.007 & n.a. & n.a. & n.a. \\
Distal M1 & $8(44 \%)$ & $9(31 \%)$ & 0.35 & 1.9 & $0.49-7.6$ & 0.37 \\
M2 & $4(29 \%)$ & $11(37 \%)$ & 0.73 & 0.51 & $0.07-3.7$ & 0.50 \\
ICA and proximal M1 & $16(47 \%)$ & $1(3 \%)$ & $<0.001$ & 28.0 & $3.4-233.8$ & 0.002 \\
Distal M1 and M2 & $12(38 \%)$ & $20(34 \%)$ & 0.82 & 1.1 & $0.37-3.1$ & 0.90 \\
\hline
\end{tabular}

P1, $p$ value between groups; P2, significance of the covariate in binary logistic regression model; CI, confidence interval; ICA, internal carotid artery; M1, middle cerebral artery segment 1; M2, middle cerebral artery segment 2 .

A current consensus statement recommends that the M1 segment should be divided into proximal and distal subsegments [9]. None of the recent randomized trials reported this distinction in their published analyses. In the earlier IMS 3 trial, 64\% of subjects in the IVT-only arm compared to $55 \%$ in the endovascular arm had good clinical outcome of an M1D occlusion [8]. The difference was not statistically significant. According to a recent retrospective study, $61 \%$ of subjects treated for M1D occlusion with MT experienced good clinical outcome and 44\% had excellent outcome [10]. These findings are in alignment with our results (Fig. 3). When we analyzed the whole M1 segment, MT borderline significantly improved the outcome (OR $=2.6$, CI 95\% 0.94-7.2, $p=0.07$ ). This is compatible with the results of recent meta-analyses $[14,15]$.

The feasibility and efficacy of MT of the M2 segment has been demonstrated in a few studies $[6,7,16]$. Successful reperfusion rates range from 78.5 to $93.3 \%$, and up to $60 \%$ of patients achieved good clinical outcome. Four of the 5 recent randomized trials reported results on the M2 segment. In the ESCAPE study, $79 \%$ of patients in the control group received IVT [3]. Only 6 patients in the intervention group had an isolated M2 occlusion and patients with occlusion of all M2 segment arteries were pooled with M1 occlusions. The authors state that the treatment effect (showing benefit from MT) was similar in location-based subgroup 
analyses but details are not provided. The SWIFT PRIME trial had 18 patients with an M2 occlusion [5]. Thirteen of them were in the MT and IVT group, and 5 in the IVT group with 53 and $40 \%$ experiencing good clinical outcome, respectively. The difference was not statistically significant. In the REVASCAT study, $78 \%$ of patients in the control group received IVT [4]. Ten patients in the MT group and 8 in the control group had an M2 occlusion whose clinical outcome was not detailed. In the MR CLEAN trial, $91 \%$ of patients in the control group were administered IVT [1]. Eighteen patients in the MT group and 21 patients in the control group had an M2 occlusion. A subgroup analysis of M2 occlusions was not reported. A metaanalysis of these trials has been published recently [14]. Pooled together, there were 51 eligible patients with an M2 occlusion in the MT group and 44 in the control group. The direction of the effect favored MT but the adjusted OR was not significant (OR $=1.28,95 \% \mathrm{CI}$ $0.51-3.21$ ). Another meta-analysis of somewhat older studies reported similar results (OR = $1.5,95 \%$ CI 0.8-3.0) [15].

The recanalization rate of an M2 segment occlusion with MT was 79\% in our study, which is comparable to the literature but not optimal. After adjusting for confounders, there was a nonsignificant trend toward worse clinical outcome in the MT group which was observed also when the M2 and M1D segments were analyzed together (Table 2). The M2 occlusion patients in the IVT-only group had milder strokes compared to the MT group (median NIHSS 10 vs. 14). However, the trend remained unchanged when patients with mild strokes (NIHSS <8) were excluded (data not shown). The reported recanalization rates of M2 occlusions with IVT are reasonably high at $44-79 \%$ and correspondingly the clinical outcomes better compared to proximal LVOs [8, 17-19]. In our data, 77\% of M2 occlusion patients treated with only IVT experienced good clinical outcome (Fig. 3), a considerably higher proportion than in the IMS 3 trial (44.5\%) [8]. This along with the somewhat suboptimal reperfusion rate in our MT group and selection of moderate and severe strokes to the MT group probably leads to too pessimistic an estimate of the efficacy of MT. That withstanding, there is no indication in our data that MT would outperform IVT in the treatment of hyperacute M2 occlusions. A very recent multicenter retrospective analysis comparing modern endovascular therapy with best medical management reported a substantial (OR 3.2, CI 2-5.2, $p<0.001$ ) advantage in favor of endovascular therapy [20]. However, this result is not directly comparable to ours because not all patients were treated with MT in the endovascular group and IVT in the medical management group. Further, patients presenting up to $8 \mathrm{~h}$ from the onset were included in the study.

Almost half of the patients in the MT group who had proximal LVO experienced excellent clinical outcome compared to only 1 patient (3\%) in the IVT-only group. Thus, it appears that surviving an ICA or M1P occlusion without any disability-causing neurological deficits essentially requires MT. This has potential implications especially in the management of patients with low prestroke mRS (0-1).

We restricted our analysis to hyperacute strokes. The time window of IVT was extended to $4.5 \mathrm{~h}$ in 2008. The efficacy of IVT deteriorates rapidly in the first few hours resulting in a sizeable number-needed-to-treat figure of 14.9 in the 3- to 4.5-h time window [21]. Considering the superior reperfusion rates of MT, one would expect the performance of MT to be at least at par with IVT in the extended time window. Extrapolating from the results of a recent meta-analysis, this appears to be the case [14].

Our observational study has several limitations: a major limitation is its retrospective nature, although the data for both groups were collected and recorded prospectively according to a preset protocol. There may be a selection of patients with more severe symptoms to MT especially in distal occlusions, and conversely those with milder symptoms may have been denied an invasive procedure. Patients that responded to IVT or experienced significant and sustained spontaneous resolution of symptoms between admission imaging and groin 
puncture were not included in the MT group, whereas those with contraindications to IVT were obviously not in the IVT-only group. The latter is the reason why the proportion of patients with atrial fibrillation was larger in the MT group. However, all these biases act to diminish the perceived efficacy of MT in relation to IVT only. Further, the somewhat small study population decreases the value of subgroup analyses. Patient level data on prestroke mRS were available only in the IVT-only group (the median mRS was 1). Several different stent retrievers or a combination of them were used in varying proportions in different clot locations based on the preference of the operator, which may lead to a bias between the proximal and distal LVOs. However, there were no significant differences in technical success between the two main devices that were used [22]. Finally, the technical details (primary stent retriever thrombectomy with a balloon-tipped guiding catheter) limit the generalizability of the results.

In conclusion, our study shows that MT is superior to IVT in achieving good clinical outcome in the treatment of hyperacute anterior circulation stroke in the most proximal occlusions, i.e. ICA or the M1P segment. In the distal M1 segment or the M2 segment, neither of these therapies clearly outperforms the other.

\section{Acknowledgments}

The authors are grateful to Jari Hakomäki, MD, for image interpretation and Ira Matkaselkä, BA, for data collection.

\section{Disclosure Statement}

All authors declare that they have no conflicts of interest relevant to this work.

\section{Funding Sources}

This study was financially supported by the Competitive State Research Financing of the Expert Responsibility Area of Tampere University Hospital (Grant 9S061).

\section{Appendix}

Imaging Parameters

In the MT cohort, the CT scans were obtained using a 64-row multidetector CT scanner (General Electric LightSpeed VCT, GE Healthcare, Milwaukee, WI, USA). Brain NCCT was performed using the parameters 120 $\mathrm{kV}$ with AUTOmA and SMARTmA technic, noise index 3.3, collimation $4 \times 5 \mathrm{~mm}, 40 \%$ adaptive statistical iterative reconstruction (ASIR), and rotation $0.5 \mathrm{~s}$. Images were obtained axially ( 0.625 -mm-thick slices), and then contiguous axial slices were reconstructed to the thickness of $5 \mathrm{~mm}$ and coronal slices to the thickness of $2 \mathrm{~mm}$. CTA was performed with helical technique using a scanning range from the aortic arch to the vertex of the skull. The imaging parameters were $100 \mathrm{kV}$, AUTOmA and SMARTmA, noise index 9, 40\% ASIR, collimation $40 \times 0.625 \mathrm{~mm}$, rotation $0.5 \mathrm{~s}$, pitch factor 0.984 . The contrast agent (iomeprol, $350 \mathrm{mg} \mathrm{I} / \mathrm{mL}$; IOMERON, Bracco, Milan, Italy) was administered via an antecubital vein with 18-gauge cannula using a double-piston power injector with a flow rate of $5 \mathrm{~mL} / \mathrm{s}$ using $70 \mathrm{~mL}$ contrast agent followed by a $50 \mathrm{~mL}$ saline flush. Automatic bolus triggering from the aortic arch was used. CTP was performed using the parameters $80 \mathrm{kV}, 250 \mathrm{~mA}, 50 \%$ ASIR, collimation $8 \times 5 \mathrm{~mm}$, and rotation $0.4 \mathrm{~s}$. 272 slices covering a range of 80 $\mathrm{mm}$ were generated in $46 \mathrm{~s}$ using alternating toggle table protocol to increase the $\mathrm{z}$-axis coverage. Contiguous slices were reconstructed to a thickness of $5 \mathrm{~mm}$ at even intervals. The contrast agent (IOMERON $350 \mathrm{mg} \mathrm{I/}$ $\mathrm{mL}$ ) was administered via an antecubital vein with an 18-gauge cannula using a double-piston power injector with flow rate of $5 \mathrm{~mL} / \mathrm{s}$ using $40 \mathrm{~mL}$ of contrast agent followed by a $40 \mathrm{~mL}$ saline flush. DSA images were 
obtained using the Artis Z angiographer (Siemens, Munich, Germany) using the parameters $102 \mathrm{kV}, \mathrm{AUTOmA}$ and SMARTmA.

In the IVT cohort, imaging was performed using two different multidetector scanners: General Electric LightSpeed 16-row (GE Healthcare, Milwaukee, WI, USA) and Philips Brilliance 64-row (Philips, Cleveland, $\mathrm{OH}$, USA). Brain NCCT was performed using the parameters $120 \mathrm{kV}, 430 \mathrm{mAs}$, collimation $12 \times 1.25 \mathrm{~mm}$, rotation $1.5 \mathrm{~s}$ (64-row) or $120 \mathrm{kV}, 320 \mathrm{mAs}$, collimation $16 \times 1.25 \mathrm{~mm}$, rotation $1 \mathrm{~s} \mathrm{(16-row).} \mathrm{Contiguous}$ slices were reconstructed to the thickness of $5 \mathrm{~mm}$ in the whole scanning range (64-row) or to the thickness of $5 \mathrm{~mm}$ in the skull base and $7.5 \mathrm{~mm}$ in the supratentorial region (16-row). CTA was performed using a scanning range extending from the $\mathrm{C} 2$-vertebra to the vertex of the skull. The imaging parameters were 120 $\mathrm{kV}, 212 \mathrm{mAs}$ (using dynamic tube current modulation), collimation $64 \times 0.625 \mathrm{~mm}$, rotation $0.75 \mathrm{~s}$, pitch factor 0.923 (64-row) or $120 \mathrm{kV}, 160 \mathrm{mAs}$, collimation $16 \times 0.625 \mathrm{~mm}$, rotation $0.8 \mathrm{~s}$, pitch factor 0.938 (16-row). Contiguous slices were reconstructed to the thickness of $0.9 \mathrm{~mm}$ with a $0.45 \mathrm{~mm}$ overlap (64-row) or to the thickness of $1.25 \mathrm{~mm}$ (16-row). The contrast agent (iobitridol, Xenetix $350 \mathrm{mg} \mathrm{I} / \mathrm{mL}$; Aulnay-sousBois, France) was administered through an antecubital 18-gauge cannula using a double-piston power injector with a flow rate of $4 \mathrm{~mL} / \mathrm{s}$ using $70 \mathrm{~mL}$ of contrast agent followed by a $50 \mathrm{~mL}$ saline flush. Manual bolus triggering was used. CTP was performed using the parameters $80 \mathrm{kV}, 200 \mathrm{mAs}$ (effective), collimation $32 \times 1.25 \mathrm{~mm}$, rotation $0.4 \mathrm{~s}$ (64-row) or $80 \mathrm{kV}, 200 \mathrm{mAs}$, collimation $8 \times 2.5 \mathrm{~mm}$, rotation $1 \mathrm{~s}$ (16-row). 120 slices covering a range of $80 \mathrm{~mm}$ were generated in $55 \mathrm{~s}$ using an alternating toggle table protocol (64-row), or 200 slices covering a range of $20 \mathrm{~mm}$ were generated in $50 \mathrm{~s}$ with a stationary gantry position (16-row). Contiguous slices were reconstructed to the thickness of $10 \mathrm{~mm}$ (64-row) or to the thickness of $5 \mathrm{~mm}$ (16-row) at even time intervals. The imaging range was positioned so that the ASPECTS levels (the level of the basal ganglia and the supraganglionic level) were always covered. The rest of the $80 \mathrm{~mm}$ range (64-row) was positioned both cranial and caudal to the ASPECTS planes with the exact balancing depending on the clinical presentation. The contrast agent (Xenetix $350 \mathrm{mg} \mathrm{I} / \mathrm{mL}$ ) was administered through an antecubital 18-gauge cannula using a double-piston power injector with a flow rate of $5 \mathrm{~mL} / \mathrm{s}$ using $60 \mathrm{~mL}$ of contrast agent followed by a $40 \mathrm{~mL}$ saline flush.

\section{Image Analysis}

NCCT, CTA, and CTP examinations were reviewed using dedicated medical imaging workstations. Parametric perfusion maps - mean transit time (MTT), cerebral blood flow (CBF), and cerebral blood volume (CBV) - were generated with the CT Perfusion 4 software that uses a delay insensitive deconvolution-based algorithm (GE Healthcare). CTA images were reviewed by examining both the raw data and maximum intensity projection images. The Alberta Stroke Program Early CT Score (ASPECTS) was assessed from admission and follow-up 24-h NCCT images and from MTT and CBV maps, and CTA was used to evaluate the occlusion site, the Clot Burden Score (CBS) and the Collateral Score (CS) as described in previous reports [23, 24]. The location of the clot was recorded based on the most proximal position of the occlusion. The M1 segment of the MCA was divided in two parts of equal length: the proximal and the distal half as described previously (M1P and M1D, respectively) [25]. The examinations were reviewed in the order NCCT, CTA, and finally CTP, paralleling that of the clinical work flow. Two radiologists assigned ASPECTS, CBS, and CS. In cases where the scoring or the assignment differed, a consensus opinion was agreed on. The reviewers were blinded to the clinical data apart from the side and nature of the acute symptoms. One radiologist measured the final infarct volumes. The boundaries of the affected areas were determined visually. Volume was calculated by multiplying the measured area with the slice thickness. TICI was evaluated and recorded prospectively to a questionnaire by the interventional neuroradiologist that performed the procedure. Intraclass correlation coefficients (ICC) between staff radiologists and an experienced neuroradiologist were calculated for ASPECTS assignments in a test sample $(n=20)$ : $\mathrm{ICC}_{\mathrm{NCCT}}=0.86, \mathrm{ICC}_{\mathrm{MTT}}=0.79, \mathrm{ICC}_{\mathrm{CBV}}=0.73$, and $\mathrm{ICC}_{\mathrm{NCCT} 24 \mathrm{~h}}=0.93$. Median interobserver agreement indices for areas and volumes were AREA $\mathrm{MTT}_{\text {: }}$ 68\%, AREA $_{\mathrm{CBV}}: 90 \%$ and VOLUME INFARCT: $80 \%$. ICC for CS was 0.87 . Cohen's kappa was 0.94 for the location of the clot and 0.68 for the collateral score (0.90 after dichotomization 0-1 vs. 2-4). 
Sillanpää et al.: Internal Carotid Artery and the Proximal M1 Segment Are Optima Targets for Mechanical Thrombectomy

Results

Baseline and admission imaging characteristics and the clinical and imaging outcomes of all patients and patients in the MT and IVT-only groups

\begin{tabular}{lcccc}
\hline Characteristic & $\begin{array}{c}\text { All patients } \\
(n=165)\end{array}$ & $\begin{array}{l}\text { MT } \\
(n=67)\end{array}$ & $\begin{array}{l}\text { IVT only } \\
(n=98)\end{array}$ & $p_{1}$ \\
\hline Age, years & $68.5 \pm 12.4$ & $67.9 \pm 9.7$ & $68.9 \pm 13.8$ & 0.60 \\
Male sex & $85(52)$ & $30(45)$ & $55(56)$ & 0.18 \\
NIHSS before treatment & $13.5[9]$ & $14(5)$ & $14(9)$ & 0.26 \\
ASPECTS score at admission NCCT & $10[2]$ & $9(2)$ & $10(2)$ & 0.34 \\
ASPECTS score at admission MTT & $4[4]$ & $2(4)$ & $5(4)$ & $<0.001$ \\
ASPECTS score at admission CBV & $8[3]$ & $7(4)$ & $8(4)$ & 0.10 \\
Onset to imaging time, min & $90 \pm 34$ & $86 \pm 64$ & $90 \pm 34$ & 0.58 \\
Clot burden score & $6[4]$ & $6(4)$ & $6(4)$ & 0.03 \\
Collateral score & $1[3]$ & $1(2)$ & $2(3)$ & 0.58 \\
Hypertension & $97(59)$ & $35(52)$ & $62(64)$ & 0.16 \\
Diabetes & $26(16)$ & $10(15)$ & $16(16)$ & 0.81 \\
Atrial fibrillation & $74(45)$ & $37(55)$ & $37(38)$ & 0.04 \\
Coronary artery disease & $45(27)$ & $11(16)$ & $34(35)$ & 0.01 \\
\hline Three-month modified Rankin scale 0-2 & $84(52)$ & $37(56)$ & $47(49)$ & 0.34 \\
Three-month modified Rankin scale 0-1 & $49(30)$ & $28(42)$ & $21(22)$ & 0.005 \\
Three-month mortality & $22(13)$ & $8(12)$ & $14(14)$ & 0.67 \\
ASPECTS score at 24-h CT & $7.5[5]$ & $8(3)$ & $7(5)$ & 0.007 \\
Total infarct volume at 24 h, cm 3 (3) & $45 \pm 75$ & $27 \pm 52$ & $57 \pm 85$ & 0.005 \\
Hemorrhagic complication at 24 h & $16(19)$ & $9(14)$ & $7(7)$ & 0.18 \\
$\quad$ Major space-occupying effect (PH2 or PHr2) & $7(4)$ & $4(6)$ & $3(3)$ & 0.44 \\
Postinfarct edema COED2 or COED3 & $43(26)$ & $14(21)$ & $29(30)$ & 0.20 \\
\hline
\end{tabular}

Data are presented as mean $\pm \mathrm{SD}, n(\%)$, or median [IQR]. $p_{1}$, p value between groups; ASPECTS, Alberta Stroke Program Early CT Score; CBV, cerebral blood volume; COED, cerebral edema; MTT, mean transit time; NCCT, non-contrast-enhanced computed tomography; NIHSS, National Institutes of Health Stroke Scale; PH, parenchymal hemorrhage; PHr, parenchymal hemorrhage remote.

\section{References}

1 Berkhemer OA, Fransen PS, Beumer D, van den Berg LA, et al: A randomized trial of intraarterial treatment for acute ischemic stroke. N Engl J Med 2015;372:11-20.

2 Campbell BC, Mitchell PJ, Kleinig TJ, Dewey HM, et al: Endovascular therapy for ischemic stroke with perfusionimaging selection. N Engl J Med 2015;372:1009-1018.

3 Goyal M, Demchuk AM, Menon BK, Eesa M, et al: Randomized assessment of rapid endovascular treatment of ischemic stroke. N Engl J Med 2015;372:1019-1030.

4 Jovin TG, Chamorro A, Cobo E, de Miquel MA, et al: Thrombectomy within 8 hours after symptom onset in ischemic stroke. N Engl J Med 2015;372:2296-2306.

5 Saver JL, Goyal M, Bonafe A, Diener HC, et al: Stent-retriever thrombectomy after intravenous t-PA vs. t-PA alone in stroke. N Engl J Med 2015;372:2285-2295.

6 Dorn F, Lockau H, Stetefeld H, Kabbasch C, Kraus B, Dohmen C, Henning T, Mpotsaris A, Liebig T: Mechanical thrombectomy of M2-occlusion. J Stroke Cerebrovasc Dis 2015;24:1465-1470.

7 Flores A, Tomasello A, Cardona P, de Miquel MA, Gomis M, Garcia Bermejo P, Obach V, Urra X, Marti-Fabregas J, Canovas D, Roquer J, Abilleira S, Ribo M; Catalan Stroke Code and Reperfusion Consortium Cat-SCR: Endovascular treatment for M2 occlusions in the era of stentrievers: a descriptive multicenter experience. J Neurointerv Surg 2015;7:234-237.

8 Demchuk AM, Goyal M, Yeatts SD, Carrozzella J, Foster LD, Qazi E, Hill MD, Jovin TG, Ribo M, Yan B, Zaidat O0, Frei D, von Kummer R, Cockroft KM, Khatri P, Liebeskind DS, Tomsick TA, Palesch YY, Broderick JP; IMS III Investigators: Recanalization and clinical outcome of occlusion sites at baseline CT angiography in the Interventional Management of Stroke III trial. Radiology 2014;273:202-210. 


\begin{tabular}{l|l}
\hline DOI: $10.1159 / 000475606$ & $\begin{array}{l}\text { C) 2017 S. Karger AG, Basel } \\
\text { www.karger.com/ine }\end{array}$ \\
\hline
\end{tabular}

Sillanpää et al.: Internal Carotid Artery and the Proximal M1 Segment Are Optimal Targets for Mechanical Thrombectomy

9 Zaidat 00, Yoo AJ, Khatri P, Tomsick TA, et al: Recommendations on angiographic revascularization grading standards for acute ischemic stroke: a consensus statement. Stroke 2013;44:2650-2663.

10 Behme D, Kowoll A, Weber W, Mpotsaris A: M1 is not M1 in ischemic stroke: the disability-free survival after mechanical thrombectomy differs significantly between proximal and distal occlusions of the middle cerebral artery M1 segment. J Neurointerv Surg 2015;7:559-563.

11 Friedrich B, Gawlitza M, Schob S, Hobohm C, Raviolo M, Hoffmann KT, Lobsien D: Distance to thrombus in acute middle cerebral artery occlusion: a predictor of outcome after intravenous thrombolysis for acute ischemic stroke. Stroke 2015;46:692-696.

12 Saarinen JT, Sillanpaa N, Rusanen H, Hakomaki J, Huhtala H, Lahteela A, Dastidar P, Soimakallio S, Elovaara I: The mid-M1 segment of the middle cerebral artery is a cutoff clot location for good outcome in intravenous thrombolysis. Eur J Neurol 2012;19:1121-1127.

13 Ding D: Endovascular mechanical thrombectomy for acute ischemic stroke: a new standard of care. J Stroke 2015;17:123-126.

14 Goyal M, Menon BK, van Zwam WH, Dippel DW, et al: Endovascular thrombectomy after large-vessel ischaemic stroke: a meta-analysis of individual patient data from five randomised trials. Lancet 2016;387:1723-1731.

15 Lemmens R, Hamilton SA, Liebeskind DS, Tomsick TA, et al: Effect of endovascular reperfusion in relation to site of arterial occlusion. Neurology 2016;86:762-770.

16 Sheth SA, Yoo B, Saver JL, Starkman S, Ali LK, Kim D, Gonzalez NR, Jahan R, Tateshima S, Duckwiler G, Vinuela F, Liebeskind DS; UCLA Comprehensive Stroke Center: M2 occlusions as targets for endovascular therapy: comprehensive analysis of diffusion/perfusion MRI, angiography, and clinical outcomes. J Neurointerv Surg 2015;7:478-483.

17 Zangerle A, Kiechl S, Spiegel M, Furtner M, Knoflach M, Werner P, Mair A, Wille G, Schmidauer C, Gautsch K, Gotwald T, Felber S, Poewe W, Willeit J: Recanalization after thrombolysis in stroke patients: predictors and prognostic implications. Neurology 2007;68:39-44.

18 Saqqur M, Uchino K, Demchuk AM, Molina CA, Garami Z, Calleja S, Akhtar N, Orouk FO, Salam A, Shuaib A, Alexandrov AV; CLOTBUST Investigators: Site of arterial occlusion identified by transcranial Doppler predicts the response to intravenous thrombolysis for stroke. Stroke 2007;38:948-954.

19 del Zoppo GJ, Poeck K, Pessin MS, Wolpert SM, Furlan AJ, Ferbert A, Alberts MJ, Zivin JA, Wechsler L, Busse O: Recombinant tissue plasminogen activator in acute thrombotic and embolic stroke. Ann Neurol 1992;32: 78-86.

20 Sarraj A, Sangha N, Hussain MS, Wisco D, et al: Endovascular therapy for acute ischemic stroke with occlusion of the middle cerebral artery M2 segment. JAMA Neurol 2016;73:1291-1296.

21 Donnan GA, Davis SM, Parsons MW, Ma H, Dewey HM, Howells DW: How to make better use of thrombolytic therapy in acute ischemic stroke. Nat Rev Neurol 2011;7:400-409.

22 Protto S, Pienimaki JP, Seppanen J, Matkaselka I, Ollikainen J, Numminen H, Sillanpaa N: TREVO and Capture LP have equal technical success rates in mechanical thrombectomy of proximal and distal anterior circulation occlusions. J Neurointerv Surg, Epub ahead of print.

23 Saarinen JT, Rusanen H, Sillanpaa N: Collateral score complements clot location in predicting the outcome of intravenous thrombolysis. AJNR Am J Neuroradiol 2014;35:1892-1896.

24 Sillanpaa N, Saarinen JT, Rusanen H, Hakomaki J, Lahteela A, Numminen H, et al: CT perfusion ASPECTS in the evaluation of acute ischemic stroke: thrombolytic therapy perspective. Cerebrovasc Dis Extra 2011;1:6-16.

25 Zaidat 00, Yoo AJ, Khatri P, Tomsick TA, von Kummer R, Saver JL, et al: Recommendations on angiographic revascularization grading standards for acute ischemic stroke: a consensus statement. Stroke 2013;44:26502663. 